\title{
SMas: A Shopping Mall Multiagent Systems
}

\author{
Javier Bajo ${ }^{1}$, Yanira de Paz ${ }^{2}$, Juan Francisco de Paz ${ }^{2}$, Quintín Martin ${ }^{3}$, \\ and Juan M. Corchado ${ }^{2}$ \\ ${ }^{1}$ Universidad Pontificia de Salamanca, Compañía 5 37002, Salamanca, Spain \\ jbajope@upsa.es \\ ${ }^{2}$ Departamento Informática y Automática, Universidad de Salamanca, \\ Plaza de la Merced s/n 37008, Salamanca, Spain \\ yanira@usal.es, fcofds@gmail.com, corchado@usal.es \\ ${ }^{3}$ Departamento Estadística, Universidad de Salamanca, \\ Plaza de la Merced s/n 37008, Salamanca, Spain \\ qmmausal . es
}

\begin{abstract}
This paper presents a multiagent model that facilitates aspects of shopping mall management, as well as increasing the quality of leisure facilities and shopping on offer. The work presented focuses on the use of a multi agent architecture, based on the use of deliberative agents that incorporates casebased planning. The architecture considers a dynamic framework, and the need to use autonomous models that are able to evolve over time. The architecture incorporates agents whose aim is to acquire knowledge and adapt themselves to the environmental changes. The system has been tested successfully, and the results obtained are presented in this paper.
\end{abstract}

Keywords: CBR, CBP-BDI, wireless multiagent system, shopping mall, SMAS.

\section{Introduction}

Agents and multiagent systems are adequate for developing applications in dynamic, flexible environments. Agents can be characterized through their capacities in areas such as autonomy, reactivity, pro-activity, social abilities, reasoning, learning and mobility. These capacities can be modelled in various ways, using different methodologies [1]. One of the possibilities is to use Case Based Reasoning (CBR). This paper presents a distributed architecture whose principal characteristic is the use of CBP agents [2, 3, 4]. These deliberative agents incorporate a reasoning CBP (Case Based Planning) engine, a variant of the CBR (Case Based Reasoning) system [5, 6]. The CBP system makes it possible for the agents to learn from initial knowledge, interact autonomously with the environment and system users, and allows it to adapt itself to environmental changes.

The aim of this work is to obtain an architecture that allows the development of multi-objective agents, which incorporate CBP reasoning mechanisms, for dynamic environments. To achieve this aim we have concentrated in a specific problem, the management of some aspects of a shopping mall, and we use an architecture that makes it possible to construct agents capable of adapting its knowledge to environmental changes. There are many different architectures for constructing

E. Corchado et al. (Eds.): IDEAL 2006, LNCS 4224, pp. 1166-1173, 2006.

(c) Springer-Verlag Berlin Heidelberg 2006 
deliberative agents and many of them are based on the BDI model. In the BDI model, the internal structure of an agent and its capacity to choose, is based on mental aptitudes. The BDI model uses the agent's beliefs as informational aptitudes, its desires as motivational aptitudes and its intentions as deliberative aptitudes. The method proposed in [7] facilitates the incorporation of CBR systems as a deliberative mechanism within BDI agents, allowing them to learn and adapt themselves, lending them a greater level of autonomy than pure BDI architecture [4]. The management of a shopping mall is a problem in a dynamic environment. Moreover several goals must be controlled, such as offers, product or service promotions, plans generation for a user profile and preferences, etc.

SMas incorporates "lightweight" agents that can live in mobile devices, such as phones, PDAs, etc. These agents make it possible for a client to interact with the SMas in a very simple way, downloading and installing a personal agent in his mobile phone or PDA. The system also incorporates one agent for each shop in the shopping mall. These agents can calculate the optimal promotions and services at a given moment. The core of the SMas is a Coordinator agent in change of the plans (routes) generation in response to a client's request, looking for the best shopping or leisure time alternatives. The agent has to take into account the client profile, the maximum amount of money that the client wants to spend, the time available and the client profile. The generation of routes must be independent of the shopping mall management, in the sense that it is not appropriate to use the same knowledge base (or all the knowledge) that the directorship controls. Only the knowledge corresponding to the offers and promotions at the moment of the client request should be used. Otherwise the client will be directed to the objectives of the shopping mall management. The agents are adapted to work in mobile devices, so they support wireless communication (Wi-Fi, Bluetooth) which facilitates the portability to a wide range of mobile devices [4]. The multiagent system can be designed using a number of methodologies: Gaia [8], AUML [9], MAS-CommonKADS, MaSE, ZEUS and MESSAGE. We have decided to opt for a combination of elements from Gaia and Agent Unified Modeling Language (AUML) for our MAS.

In the next section, we will explain the shopping mall problem that has led to most of this research. In the third section we will describe the wireless multiagent system developed, paying special attention to the Planner agent. Finally, some preliminary results and the conclusions will be presented.

\section{Shopping Mall Problem}

The Mall has become one of the most prevalent alternatives to traditional shopping [10]. A shopping mall is a cluster of independent shops, planned and developed by one or several entities, with a common objective. The size, commercial mixture, common services and complementary activities developed are all in keeping with their surroundings [10]. Our aim is to develop an open system, capable of incorporating as many agents as necessary, agents that can provide useful services to the clients not only in this shopping centre, but also in any other environment such as the labor market, educational system, medical care, etc. The system provides mechanisms for free easy data consulting. A user will be able to gain access to 
commercial (shopping and sales) and leasing time information (entertainment, events, attractions, etc.) by using their mobile phone or PDA. Mechanisms for route planning when a user wants to spend their time in the mall will also be available. Moreover, it provides a tool for advertising offers (a commercial manager will be able to make his offers available to the shopping mall clients), or provides a tool to the shopping mall management team in order to contact commercial managers or shopping mall clients, providing an interaction between users interested in the same topics.

The SMas incorporate agents based on a multi objective architecture, such as BDI, which incorporates Case Based Planning mechanisms as a reasoning engine and includes dynamic replanning algorithms. The CBP uses the CBR concepts to reason and create plans. Moreover, the incorporation of a dynamic replanning technique (Most-Re-plan-able Intention) offers the possibility of replanning at time of execution $[5,2]$ thereby ensuring that every client will use optimal plans at execution time. The use of artificial intelligence models makes it possible to take decisions about the optimal route for a user with a given profile and his preferences at the moment when the suggestion is requested.

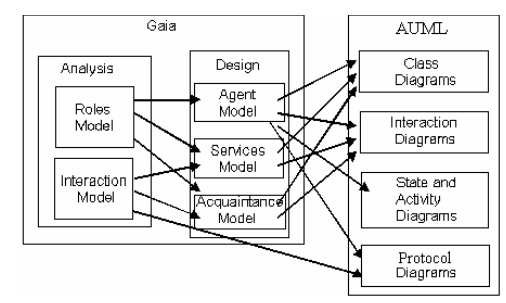

Fig. 1. Methodology used in the development process

\section{SMas System Architecture}

The option chosen to define an appropriate analysis and design methodology for the problem to be resolved is one that combines Gaia [8] and AUML [9], in an attempt to take advantage of both. Through Gaia it is possible to make an analysis of the problem using organizational criteria and a later design. After applying Gaia, the result consists of a design at the elevated abstraction level. At this point the Gaia design is transformed so that AUML techniques can be applied. Figure 1 illustrates the paths followed in order to obtain the different models used. It shows how Gaia is used initially in order to obtain an analysis and high level design and then AUML is used in order to obtain a detailed, low level design.

Studying the requirements of the problem we have come to the conclusion that we need nine roles: The Communicator role manages all the communications of a client. The Finder role looks for near devices. The Profile Manager role obtains a client profile. The Store operator is in charge of manage the store: data base operations on stored products. Moreover monitors the products shortage, in order to prevent desupply. The Promotions manager role controls the shells in each shop, as well as the promotions that every shop offers to its clients. The Clients Manager role deals with the client profiles management and controls the connected clients at a given 
moment. The Analist role carries out periodic evaluations on shells, promotions and surveys data trying to provide a good quality service. The Incidents Manager role manages incidents, such as sending advices, or solving a wide range of problems. The Planner role is the most important role in our system. The Planner creates a route printing the most suitable shops, promotions or events to the client profile and available resources at one particular moment. As can be seen in Figure 2, the Incidents Manager role is composed of responsibilities, permissions, activities and protocols. The Incidents Manager is authorized to read and change the Incidents DB, and it is responsible for the incidents management, product orders and sending advices. Besides it must maintain a successful connection with the Incidents DB.

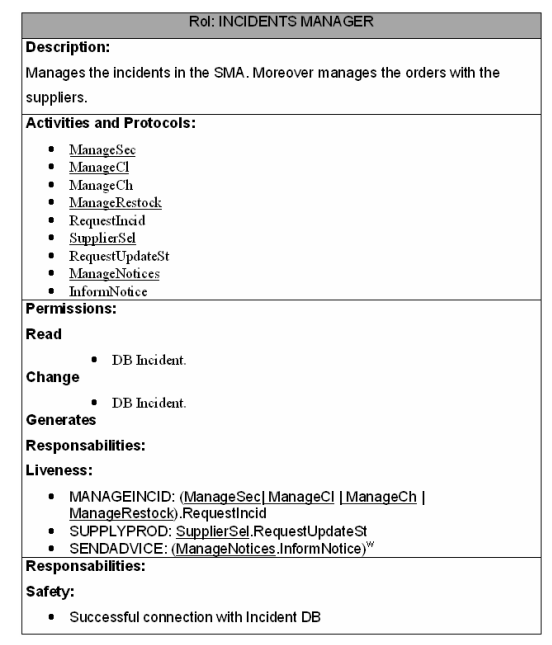

Fig. 2. Gaia roles model: Incidents Manager role

As far as interaction model is concerned, the dependences and relations between roles are described. Each interaction in which two roles are involved requires protocols (described in the roles model). In the SMA presented in this work the next protocols have been considered: RequestPromotionsData, SolveConsult, StoreProducts, AlertShortage, OrderSupplier, InformProductsState, InformPromotionsState, SolveIncident, SolveSuggestion, Notify. Figure 3 shows the interaction diagram that represents the interaction between the Analist and the Store Manager roles when the Analist requests a promotion inform.

Traditional techniques of software engineering are not followed in terms of detailing the analysis to the extent that a direct implementation can be made. Instead, the level of abstraction is reduced so that traditional techniques can be applied. In the design process three models are considered: agent model, services model and acquaintance model [8]. As we can see in Figure 4, the agent model shows the types of agents that are going to appear in the system, as well as the number of instances for each agent type that can be executed within the execution time. For example agent Store plays the Promotions Manager and Store Operator roles. 


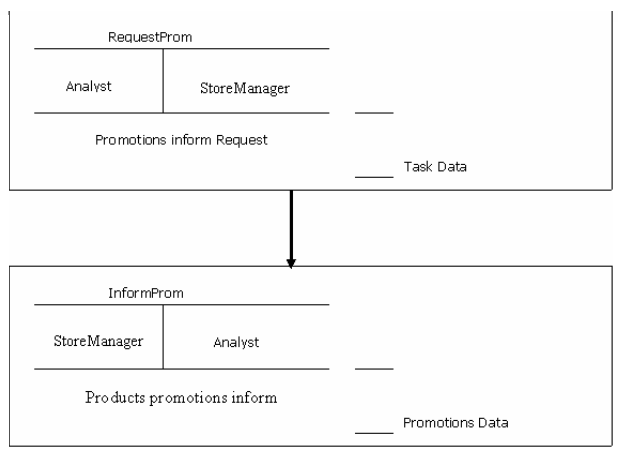

Fig. 3. Gaia interaction model: interaction RequestPromotionsData
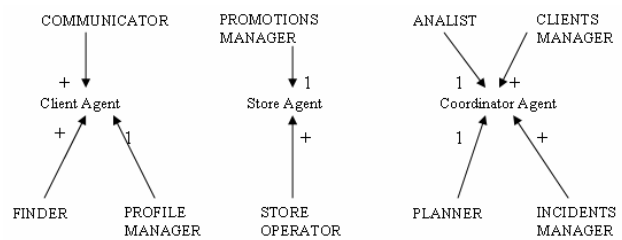

Fig. 4. Gaia agent model for the shopping mall problem

Our proposal deals with how to use the high level analysis and design obtained through the Gaia methodology to achieve a low level AUML design, with enough detailed for an implementation to be carried out. There are three concepts that vary slightly with respect to their meaning in Gaia and AUML: role, service and capability [9]. The AUML design provides class diagrams for each agent, collaboration or sequence diagrams for each interaction, state and activity diagrams to represent internal states and protocol diagrams to model communicative acts [9].

In our system a CBP agent is used, the Coordinator agent. It is an agent that deals with multiple objectives derived from the tasks of coordinating all the shops in the shopping mall, client management and its main task, planning and optimization of routes. The routes and promotions proposed to a client consider the client profile and his resources (money and time) at the moment of the route request. In the Figure 5 it is possible to observe that the Coordinator agent is able to generate routes, analyze shell and promotion data, manage incidents and manage clients at the same time. To solve the problem of routes generation the Coordinator uses an innovative planning mechanism: the case based planning. CBP provides the agent with the capabilities of learning and adaptation to the dynamic environment. Moreover, the Coordinator will be able to apply a dynamic replanning technique, the MRPI (Most RePlan-able Intention), which allows the agent to change a plan at execution time when an incident happens [3]. The Coordinator agent has seven capabilities and offers three services that are available to the rest of the agents of the SMA. It is necessary play especial attention to the Update, KBase y VCBP capabilities. The reason is that these capabilities implement the reasoning cycle of the CBP system. The Update capability implements the retrieve and retain stages, while the KBase capability implements the 
reuse stage and the capability VCBP the revise stage. The VCBP capability is also in charge of dynamic replanning task. The AUML roles are obtained through the liveness properties described in the Gaia role model role.

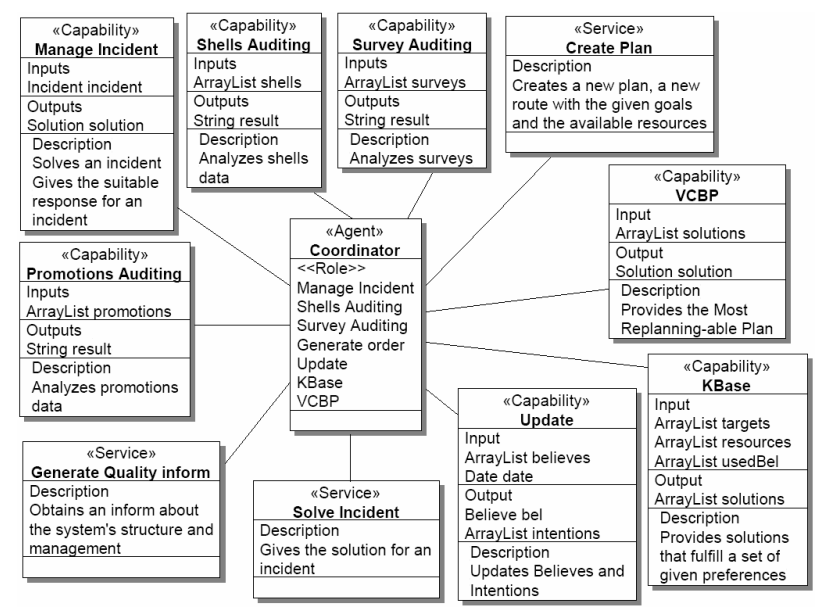

Fig. 5. Coordinator agent class diagram

Finally, to complete the AUML design, the collaboration and sequence diagrams are obtained. The protocol diagrams represent the communicative acts in the system. Once the design is finished, the implementation is carried out. The platform chosen is Jadex, a Jade [11] add-on that incorporates the BDI model to the Jade agents.

\section{Results and Conclusions}

The previously described system was tested at the Tormes Shopping Mall in the city of Salamanca during 2005. During this period of time, the multiagent system has been tuned and updated, and the first autonomous prototype started to work in October 2005. Although the system is not fully operational and the aim of the project is to construct a research prototype and not a commercial tool, the initial results have been very successful from the technical and scientific point of view. Figure 6 presents two screen shots of the User agent. Figure 6 shows the form for introducing personal data. It also shows the route generated for a client that wants buy clothes and take coffee. The fundamental concept when we work with a CBR system is the concept of case, and it is necessary to establish a case definition. A case in our problem, managed by the Coordinator agent, is composed of the attributes described in Table 1. Cases can be viewed, modified and deleted manually or automatically by the agent (during its revision stage). The agent plans (intentions) can be generated using different strategies since the agent integrates different algorithms.

SMas has been tested during the last three months of 2005 and the results have been very accurate. The system implementation has involved an increase in benefits due to the generation of automatic promotions. The e-commerce techniques [10] have 
facilitated custom-designed services for the clients. A user can easily find the products he is interested in, spend his leisure time in a more efficient way and make contact with other clients with whom he can share hobbies or opinions. So the degree of client satisfaction has been improved as observed in the surveys. The percentage of the sale of promotional products has grown over the total. The fundamental reason is that clients have instantaneous information about the products the agent thinks they are interested in, and the information is very accurate and customized.
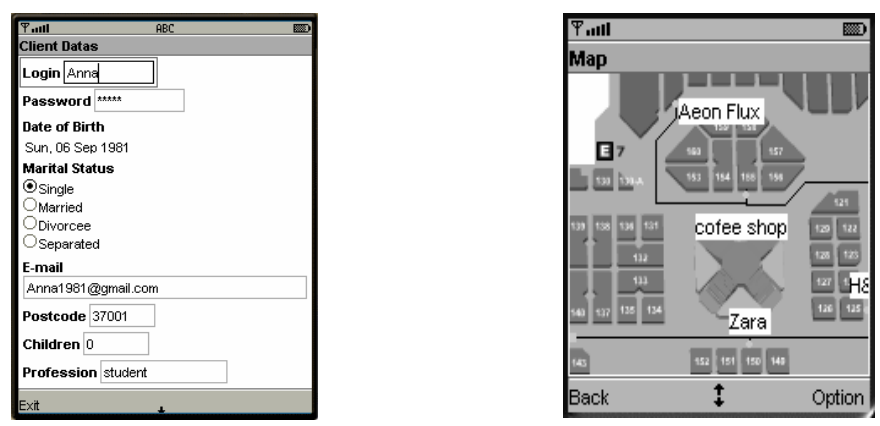

Fig. 6. Screen shots for user profile and inform route

Table 1. Case fields

\begin{tabular}{ll}
\hline Case Field & Measurement \\
\hline CLIENT & Client personal profile (ClientProfile) \\
MONEY & Money to spend (Money) \\
TIME & Available Time (Time) \\
INIT & User initial location (Location) \\
PREF & User preferences (Preference) \\
\hline
\end{tabular}

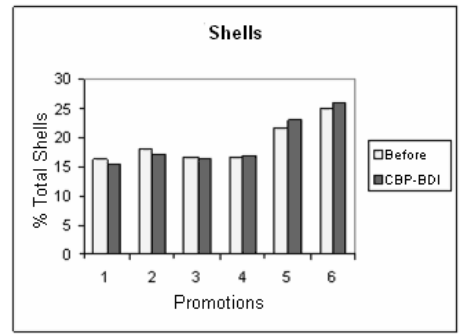

Fig. 7. Shell promotional products and shell total products

The increase in promotional products sold can be seen in Figure 7. Figure 7 shows how new promotions are launched, and the increased percentage in promotional products sold in comparison with the promotional products sold using traditional 
commercial techniques carried out the year before. We can observe that at the beginning, the results obtained with the multiagent system were worse than traditional techniques. However, as the system obtained more information about client profiles, products and habits, so the knowledge it obtained became more suitable and it was able to create optimal plans. Moreover the clients also needed some time to get used to the new system.

Acknowledgements. This work has been partially supported by the MCYT TIC200307369-C02-02 and the JCYL-2002-05 project SA104A05.

\section{References}

1. Wooldridge, M. and Jennings, N. R. (1995) Agent Theories, Architectures, and Languages: a Survey. In: Wooldridge and Jennings, editors, Intelligent Agents, SpringerVerlag, pp. 1-22.

2. Corchado J. M., Pavón J., Corchado E. and Castillo L. F. (2005) Development of CBR-BDI Agents: A Tourist Guide Application. 7th European Conference on Case-based Reasoning 2004. Lecture Notes in Artificial Intelligence 3155, Springer Verlag. pp. 547-559.

3. Glez-Bedia M. and Corchado J. M. (2002) A planning strategy based on variational calculus for deliberative agents. Computing and Information Systems Journal. Vol 10, No 1, 2002. ISBN: 1352-9404, pp. 2-14.

4. Glez-Bedia M., Corchado J. M., Corchado E. S. and Fyfe C. (2002) Analytical Model for Constructing Deliberative Agents, Engineering Intelligent Systems, Vol 3: pp. 173-185.

5. Bajo J. and Corchado J.M. Evaluation and monitoring of the air-sea interaction using a CBR-Agents approach Proccedings of the 6th Internacional Conference on Case-based Reasoning, ICCBR'05 pp. 50-62. Springer Verlag. (2005)

6. Corchado J. M. and Laza R. (2003). Constructing Deliberative Agents with Case-based Reasoning Technology, International Journal of Intelligent Systems. Vol 18, No. 12, December. pp.: 1227-1241

7. Bratman, M.E. (1987). Intentions, Plans and Practical Reason. Harvard University Press, Cambridge, M.A.

8. Wooldridge, M. and Jennings, N. R. and Kinny, D. (2000) The Gaia Methodology for Agent-Oriented Analysis and Design. Journal of Autonomous Agents and Multi-Agent Systems, 3 (3). pp. 285-312.

9. Bauer, B. and Huget, M. P. (2003) FIPA Modeling: Agent Class Diagrams.

10. Adams, F.G. (2003): The E-Business Revolution \& the New Economy: E-conomics after the Dot-Com Crash. South-Western Educational Pub.

11. Bellifime, F. Poggi, A. and Rimasa, G. (2001) JADE: a FIPA2000 compliant agent development environement. Proceedings of the $5^{\text {th }}$ international conference on autonomous agents (ACM). 